\title{
Ai Yasunaga \\ Paul Valéry dans ses derniers jours : au miroir de Voltaire
}

Résumé: Notre article entend mettre en lumière la vie et la pensée de Paul Valéry (1871-1945) durant ses derniers jours, notamment en nous référant à Voltaire, qui a fasciné Valéry vers la fin de sa vie. Dans des circonstances difficiles sur le plan politique, matériel, physique et même amoureux, Valéry âgé a trouvé en Voltaire une sorte de fil conducteur, l'incarnation de l'esprit de la liberté. Le 10 décembre 1944, moins de quatre mois après la Libération de Paris, Valéry prononce à l'occasion du 250 anniversaire de la naissance de Voltaire un discours qui exalte l'espoir de la liberté de l'esprit devant un public attendant l'avènement d'une nouvelle époque. Ce discours a suscité auprès du grand public une profonde et vaste émotion. À travers l'analyse de la vie et de l'écriture de Valéry, nous examinerons l'image d'un poète sous la Troisième République.

Mots clés: Paul Valéry, Voltaire, vie et pensée de l'auteur, image du poète sous la Troisième République, liberté de l'esprit

Notre article entend mettre en lumière la vie et la pensée de Paul Valéry (18711945) durant ses derniers jours, notamment en nous référant à Voltaire (16941778), le célèbre philosophe français des Lumières, qui a fasciné Valéry sur la fin de sa vie, alors que celui-ci vivait dans des circonstances difficiles. La volumineuse biographie de Valéry écrite par Michel Jarrety (Jarrety 2008) nous détaille minutieusement ses difficultés politiques, matérielles, physiques et amoureuses pendant cette période.

Il est à noter que dans la situation sans issue où se trouvait Valéry, atteint de violents maux d'estomac, la figure de Voltaire a pris une signification particulière. À en croire André Gide (1869-1951), qui s'est plusieurs fois rendu à son chevet pendant ses derniers jours, y compris le jour même de sa mort, le dernier livre qu'il ait vu entre les mains de Valéry était l'Essai sur les mœurs et l'esprit des nations (1756) de Voltaire ( $c f$. Jarrety 2008, 1208). Quelle est la signification de sa vénération pour Voltaire jusqu'à son dernier souffle ? Nous répondrons à cette question à travers les données biographiques et les analyses minutieuses trouvées dans ses textes sur Voltaire. 


\section{Les « autres » pour Valéry}

Avant d'aborder ce thème de la figure de Voltaire durant les derniers jours de Valéry, il importe de montrer l'importance primordiale de l'existence des « autres » chez Valéry. Il écrit dans ses Cahiers «Voilà un échiquier où tout le monde a mis la main, excepté moi » (Valéry 1973), en citant quelques noms qui ont marqué et orienté sa vie. Cette expression aphoristique pourrait susciter le désaccord de ceux qui considèrent Valéry comme une image plus ou moins statique, par exemple de « Moi pur », notion proposée par Valéry lui-même. Mais au contraire de ce préjugé sur le « Moi dur » de Valéry, il s'est souvent inspiré des autres, vivants ou morts. Pour le jeune Valéry, Rimbaud, Mallarmé et Richard Wagner étaient à la fois ses modèles et ses maîtres, mais également les objets de son adoration et les causes de son désespoir. Ces trois figures ont guidé le jeune Valéry dans les tâtonnements le menant vers la poésie dont il rêvait. Valéry avait décidé de rejeter « des choses vagues » (selon son expression), y compris l'amour et la littérature, après le drame de sa conversion dans la nuit de Gênes en 1892. Mais avec la publication en France des manuscrits de Léonard de Vinci, la figure de Vinci est également devenue un modèle qui a suscité l'écriture de ses volumineux Cahiers.

C'est « l'autre », en l'occurrence Huysmans, qui lui a permis de faire une carrière administrative. Ce sont aussi « les autres », ici Mallarmé et Degas, qui lui ont présenté celle qui allait devenir son épouse ; c'est encore " l'autre », cette fois Pierre Louÿs, qui lui a conseillé, en 1912, de publier ses poèmes de jeunesse après un silence poétique de près de vingt ans. La révision de ces poèmes de jeunesse est l'occasion pour lui de créer une nouvelle poésie, qui se cristallise dans $L a$ Jeune Parque, parue en 1917. Le succès exceptionnel de cette œuvre a aussitôt fait de Valéry une sorte d' « orateur officiel » de la Troisième République. Les intellectuels français espéraient qu'il réponde à la nécessité d'effectuer une analyse pertinente de la vie culturelle en France et permette une meilleure compréhension de leur situation en la resituant dans un contexte plus vaste. Valéry a joué ce rôle, non sans ironie.

Ainsi, on pourrait dire que Valéry, devenu le grand intellectuel de la République après le succès de La Jeune Parque en 1917, est une figure qui représente l'inconscient culturel désirable de la République française. Ce mécanisme selon lequel Valéry s'est reconnu très rapidement comme l'homme de lettres représen-

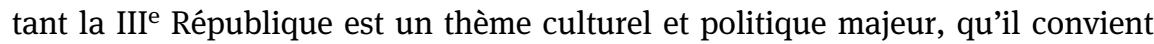
d'élucider. La publication de La Jeune Parque se situe à l'époque du choc collectif de la Grande Guerre. Valéry, dégagé de ses obligations militaires à cause de sa constitution chétive, a voulu s'engager en composant La Jeune Parque, véritable monument de la langue française. C'était sa façon à lui de s'engager durant les temps difficiles que traversaient la France et l'Europe. 
Quant à Voltaire, il était la figure la plus vénérée du lettré qu'était Paul Valéry, cet intellectuel typique de la III ${ }^{e}$ République. Il a aussi été son dernier soutien moral.

\section{La vie de Valéry : de l’Occupation à la Libération}

Les dernières années de Valéry coïncident avec les années de l'Occupation allemande, qui commence en 1940 et se termine à la Libération en 1944. Ces temps sombres ont obligé Valéry à opérer certains choix politiques et moraux. Durant cette période, sa vie comprend trois volets. Le premier est bien sûr son activité d'écrivain. Le deuxième est sa vie d'homme public : Valéry occupait diverses fonctions officielles, en sa qualité de membre de l'Académie française, de professeur de Poétique au Collège de France, et de directeur du Centre universitaire méditerranéen. Il était en outre membre du Comité National des Écrivains et du Pen Club. La préparation de ses cours, notamment au Collège de France, prenait une place importante dans sa vie, souvent ressentie comme une source de prestige, mais aussi comme un fardeau sur ses vieux jours. Le troisième volet de sa vie pendant ces années est constitué par sa liaison avec sa dernière maîtresse, Jean Voilier (pseudonyme de Jeanne Loviton). Valéry, déjà âgé, a tenté de vivre pleinement chacune des ces trois vies avec une énergie étonnante, comme s'il résistait à l'approche de sa mort.

Examinons à présent le premier volet. Au centre de la vie de l'écrivain se situent ses Cahiers, écrits non pas pour être nécessairement publiés, mais pour établir les fondements de ses idées et de sa poésie. Parallèlement à la rédaction de ses Cahiers, Valéry a préparé et révisé Mon Faust, sous la forme d'un dialogue, ainsi qu'un poème intitulé « L'Ange ». Les demandes de discours et d'articles ne suscitent pas véritablement l'intérêt de Valéry. L'impulsion de son écriture est toujours le résultat de l'entrecroisement d'incitations publiques et de sa propre lucidité. C'est ce que révèle notamment la rédaction de ses Cahiers. Mais le discours qui lui est demandé pour célébrer le $250^{\mathrm{e}}$ anniversaire de la naissance de Voltaire, en 1944, paraît lui donner l'occasion de réfléchir à une figure éminente représentant l'idée de « la liberté de l'esprit », un sujet qui le préoccupait depuis des années.

Sous l'Occupation, les publications étaient étroitement contrôlées par les $\mathrm{Na}$ zis. Il était même difficile de se procurer du papier pour la publication. Durant cette période, Valéry n'a pas eu à s'opposer directement aux Nazis, mais il « résistera » malgré tout, en refusant par exemple la nomination de Drieu La Rochelle (1893-1945) au poste de directeur de la Nouvelle revue française, qui se voulait l'expression d'une littérature honnête et authentique en France. Son refus sera catégorique. Ce durcissement a donné aux membres du comité de rédaction de la 
$N R F$ et à ses amis littéraires l'impression qu'il devenait « résistant ». Après la Libération, Valéry se consacre à la liquidation du Pen Club et aux nominations à l'Académie française. À propos de sa vie sous l'Occupation, il a eu ce mot :

Je n’ai pas fait beaucoup de résistance. Un peu à l'académie. (Note sur 1945, BnF, non coté)

Le troisième volet de sa vie est celui de ses amours amères avec Jean Voilier (1903-1996). Pendant une dizaine d'années, Valéry a entretenu une relation étroite avec cette intellectuelle, ancienne avocate et patronne d'une maison d'édition. Ils s'étaient rencontrés lors d'un cours donné par Valéry au Collège de France en 1937. Valéry confessera la douleur que lui procure la vieillesse, déplorant la triste distance qui s'installe entre eux. Les citations qui suivent illustrent la face cachée de ce grand poète et intellectuel représentant la III ${ }^{\mathrm{e}}$ République.

Mes matins ne sont plus ce qu'ils étaient naguère, ces matins de cristal, presque douloureux parfois, à force d'acuité et d'abondance. Et la chute du jour est chaque jour ma chute. J'ai souvent l'impression pénible de quelque chose dure et immobile dans ma tête. Et il faut que je travaille plus que jamais. (Valéry 1942)

Je suis comme sur un sommet suiffé et je coule à chaque instant dans un somme-songe. Ce sont des chutes insensibles. Travail essoufflé. Comme un homme épuisé qui fait 3 pas, puis s'arrête et tombe sur un banc. Ainsi mon esprit. (Cahiers, CXXVI, 705)

Je suis désagrégé, dévasté. Image de la haute tige rompue qui ne se trouve plus que le poids de son néant. (Ibid.)

Le $1^{\text {er }}$ avril 1945, Jean Voilier décide d'annoncer à Valéry son mariage avec Robert Denoël, en lui promettant une possibilité d'établir un nouveau lien plus fécond. Cette explication ne le convainc guère. L'annonce lui fait véritablement l'effet d'un « coup de hache » (Jarrety 2008, chap. LVI). Après ce choc, sa santé et son moral deviennent de plus en plus précaires, et ses douleurs gastriques ne le quitteront plus.

\section{Discours sur Voltaire}

Le 10 décembre 1944, à l'occasion de la célébration du 250 anniversaire de la naissance du philosophe, Valéry prononce dans le grand amphithéâtre de la Sorbonne un discours sur Voltaire que Michel Jarrety commente ainsi dans sa récente édition des Euvres de Valéry :

Pour la première fois depuis la chute de régime de Vichy, mais aussi la dernière [Valéry] renoue avec le rôle d'orateur quasi officiel de la République qu'il avait déjà joué durant l'entre-deux-guerres. (Valéry 2016, 1283) 
Ce discours est prononcé dans une atmosphère solennelle, après une Marseillaise suivie de quelques mots du critique Émile Henriot et de Henry Wallon, psychologue spécialiste de l'éducation des enfants, présent en sa qualité de secrétaire général de l'Éducation nationale. La commémoration est un moment de rassemblement pour la communauté nationale sortant de l'Occupation, au seuil d'une nouvelle période.

Dans ce discours, Valéry ne mentionne pas directement les textes de Voltaire. Il présente la figure de Voltaire comme un écrivain engagé qui s'enracine dans l'âme des Français. Valéry ajoute que Voltaire est « spécifiquement français, inconcevable sous d'autres cieux », " sous un autre ciel que celui de Paris » (Valéry 2016, 1286). Valéry met en exergue l'existence de Voltaire, qui provoque encore des réactions opposées 250 ans après sa mort. Selon lui, Voltaire est « indéfiniment actuel » (ibid.). En fait, en préparant ce discours, Valéry note dans ses Cahiers :

Ce n'est pas là ce que je pense de Voltaire, mais ce que j'ai pensé qu'il fallait que j'en dise, vu les circonstances. Ce que j'en pense - ou plutôt ce que j'en penserais en toute liberté - je n'en sais encore rien. (Cahiers, XXIX, 315)

Comme nous pouvons le remarquer, Valéry était très conscient du « temps présent ", et il évoque Voltaire comme l'incarnation même de la liberté de l'esprit, « l'inventeur incontestable de cette fameuse et impérieuse notion toute française du Classique » (Valéry 2016, 1287) selon ses propres termes. Cette notion de « liberté de l'esprit » était aussi le sujet primordial de Valéry, en charge des affaires du comité intellectuel de la Société des Nations. Il conclut à propos de la figure de Voltaire qu'il est une sorte de « héraut de la littérature engagée » (Valéry 2016, 1283). On peut imaginer que présenter la notion de « liberté de l'esprit » peu de temps après la Libération a probablement suscité une émotion profonde et vive chez ses auditeurs. On peut également citer un extrait de son article intitulé « Respirer... », paru dans le journal Le Figaro le 26 août 1944 :

La liberté est une sensation. Cela se respire. L'idée que nous sommes libres dilate l'avenir du moment. Elle fait s'éployer à l'extrême dans nos poitrines je ne sais quelles ailes intérieures dont la force d'enlèvement enivrant nous porte. (Valéry 1948, 397-398)

On note ici une idée de liberté de l'esprit exprimée dans un langage fondamentalement corporel. Dans cet article paru quatre mois avant le discours sur Voltaire, la notion de liberté est, plus encore qu'une simple sensation, un choix résultant d'une volonté délibérée.

Le classicisme évoqué lors du discours commémoratif sur Voltaire est comparable à celui de Valéry lui-même, qui se méfiait de la simple diffusion de la passion ou de la création directe sous l'effet de l'inspiration sans maîtrise. Pour lui, le 
classicisme est maîtrise. Voltaire, dont la vie va du siècle de Louis XIV jusqu'à une dizaine d'années avant la Révolution, jette le grand pont de l'écriture et est conçu comme le grand modèle de toute personne d'envergure humaine.

Il est à noter que Valéry apprécie en particulier le dernier tiers de la vie de Voltaire, où celui-ci s'est métamorphosé en ami et défenseur du genre humain :

À l'âge où les carrières communément s'achèvent, où il était en possession de toute la renommée que les Lettres peuvent donner à quelqu'un, admiré de toutes parts, riche, n'ayant plus qu'à jouir de cette universalité légère qui se jouait dans l'atmosphère encyclopédique de son temps, si enivrant pour l'intelligence, dont ce temps fut l'âge d'or, voici qu'il se transforme en celui que nous célébrons aujourd'hui. S’il fût mort à 60 ans, il serait à présent à peu près oublié, et nous ne serions pas solennellement ici pour rendre hommage à l'auteur de Zaïre et de la Henriade. (Valéry 2016, 1292)

Valéry n'enferme pas Voltaire dans les palais de l'histoire littéraire. Le nom de Voltaire est un symbole de la " comédie de l'intellect », celui d'un vrai combattant cherchant la liberté, qui s'oppose aux infâmes et aux intolérances.

À la fin de son discours commémoratif, Valéry se confronte à son époque et aux misères du monde, évoquant la figure d'un éventuel « Voltaire gigantesque » et interpellant ses auditeurs :

Où est le Voltaire, la voix qui s'élèvera aujourd'hui ? Et quel Voltaire gigantesque, à la mesure du monde en feu, faudrait-il pour accuser, maudire, ravaler le forfait énorme et planétaire aux proportions d'un crime crapuleux ? (Valéry 2016, 1298-1299)

Ce discours sur Voltaire est, comme le dit Michel Jarrety, un « tragique bilan de la guerre » et un " constat de faillite de cette politique de l'esprit que lui-même, quinze ans durant, a défendu dans le cadre de la Société des Nations » (Valéry 2016, 1189).

Le discours ayant été diffusé en direct à la radio, Valéry a reçu des lettres d'auditeurs émus par ses propos. Ce retentissement incite Valéry à parler de Voltaire dans ses cours de Poétique au Collège de France.

\section{Dernier visage}

Le choix de Voltaire comme sujet de cours au Collège de France nous parait dû en partie au hasard et en partie à la nécessité : la demande d'un discours à l'occasion de la célébration du $250^{\mathrm{e}}$ anniversaire de la naissance du philosophe arrive à Valéry pendant la période de préparation de son cours pour la nouvelle année au Collège, mais il existait certainement des liens entre la vie et l'écriture de Voltaire et celles de Valéry. Avant tout, c'est l'idée de la liberté de l'esprit qui unit ces deux 
écrivains. Valéry tente de suggérer cette idée devant les auditeurs de son cours de Poétique au Collège de France, par un simple épisode inattendu, mais reflétant la mentalité de l'époque.

Ce cours, que je reprends une fois encore, se rouvre sous un jour bien heureusement différent de beaucoup d'autres qui le précédèrent. Un régime d'arrière-pensées se consumant, une contrainte générale du discours a cessé de s'exercer et je puis répéter ce que j'ai dit il y a quelques mois à un officier de l'armée qui, m'arrêtant devant la grille de ce Collège, me demanda si c'était là un Musée. Je lui répondis : « - C’est une école. » « - Et qu'est-ce que l'on enseigne dans cette école ? » " - Il serait trop long de vous l'expliquer. Je vous dirai seulement ceci : C'est une maison où la parole est libre. » Sur quoi il me salua et je le saluai. (Dossier Valéry 1944)

Valéry fascinait les auditeurs réunis pour l'écouter dans la salle de cours du Collège. Dans une atmosphère de soulagement et d'espoir, il a continué ses cours, mais en mai 1945, il est obligé de quitter la chaire du Collège à cause de l'aggravation de l'état de santé.

Bien que le docteur lui ait ordonné de garder le lit et d'observer un repos complet, Valéry désobéit en installant un petit bureau dans sa chambre pour lire et écrire. C'était pour lui une question de survie.

Dans la biographie de Valéry rédigée par Jarrety, on relève des dialogues avec une religieuse nommée Claire Vanier. La foi de Jeannie, l'épouse de Valéry, était trop profonde pour qu'elle ne se montre pas désireuse de voir son mari finir sa vie dans la paix de l'Église. Mais l'attitude de celui-ci envers le catholicisme a été ambiguë jusqu'à son dernier souffle. Il n'a jamais admis l'existence de Dieu. Son attitude à l'égard de la religion nous fait penser à celle de Voltaire, à mi-chemin entre le théisme et l'athéisme.

Paul Valéry meurt le 20 juin 1945, en dépit des efforts des médecins et du soutien de sa famille et de ses amis, et malgré les injections de pénicilline envoyées des États-Unis grâce aux bons offices du général De Gaulle, à propos duquel Valéry a écrit :

Difficile de faire l'analyse chimique qui discernerait l'homme, le militaire et le politique. Il me semble cependant qu'il a la concentration de celui qui joue une partie des plus compliquées. Il y a bien des cartes dans le jeu actuel. (Cahiers, XXIX, 11-12)

C'est De Gaulle qui décida d'organiser des funérailles nationales pour Paul Valéry. En un sens, il s'agissait d'un geste solennel en faveur de l'unité française, encore bien fragile. Ces obsèques furent l'occasion d'une grande mise en scène, avec la projection de deux faisceaux lumineux en forme de V. Citons également un extrait de l'hommage prononcé par le ministre de l'Éducation nationale lors de la cérémonie : 
La France se reconnaît en lui, car elle sait que jamais, au plus profond de la souffrance, ou dans l'ivresse du triomphe, ou dans l'âpre reconquête de sa force, elle ne cessera d'écouter sa conscience, et de s'éclairer à la lumière de l'intelligence. (BO $\mathrm{n}^{\circ} 48$, supplément)

C'est ainsi que Valéry, qui voyait en Voltaire une figure éminemment française et un symbole de la liberté, est devenu lui aussi l'icône culturelle et politique de la France après la Libération.

\section{Bibliographie}

[BO] Bulletin officiel. Paris : Ministère de l'Éducation nationale. [Cahiers] Valéry, Paul. Cahiers. Paris : CNRS Éditions, 1957-1961. Dossier Valéry. Paris : Collège de France, 1944 [BnF Naf 19204]. Jarrety, Michel. Paul Valéry. Paris : Fayard, 2008.

Valéry, Paul. Lettre inédite à Jean Voilier (3 novembre 1942). Sète, Médiathèque de Sète. Valéry, Paul. «Vues » (Le Figaro, 26 août 1944). Paul Valéry. Vues. Paris : La Table ronde, 1948. Valéry, Paul. Euvres complètes. Tome 1. Paris : Gallimard, 1973.

Valéry, Paul. Euvres. Tome 3. Michel Jarrety (éd. et notes). Paris : Le Livre de Poche, « La Pochothèque », 2016.

Born in Hiroshima in 1965, Ai Yasunaga is a professor of Modern French Literature at Shizuoka University, Japan. She is the author of « Gladiator » comme signe intime : La problématique de l'entraînement chez Paul Valéry, and has published Japanese translations of Patrick Modiano's Livret de famille, and Philippe Pons' Misère et crime au Japon. 